Original

\title{
Conditioned medium from rat dental pulp reduces the number of osteoclasts via attenuation of adhesiveness in osteoclast precursors
}

\author{
Hironori Mori1,2), Kazunori Hamamura1), Shoyoku Yo1,2), Kosuke Hamajima1,2), \\ Kenji Ootani3), Masaki Honda4), Kyoko Ishizuka1), Hisataka Kondo'), \\ Kenjiro Tanaka1), Daisuke Kodama ${ }^{5)}$, Takao Hirai6), Ken Miyazawa'2), \\ Shigemi Goto ${ }^{2)}$, and Akifumi Togari1) \\ 1)Department of Pharmacology, School of Dentistry, Aichi Gakuin University, Nagoya, Japan \\ 2)Department of Orthodontics, School of Dentistry, Aichi Gakuin University, Nagoya, Japan \\ 3)Advanced Cell Technology and Engineering Ltd, Tokyo, Japan \\ 4)Department of Oral Anatomy, School of Dentistry, Aichi Gakuin University, Nagoya, Japan \\ 5)Laboratory of Neuropharmacology, School of Pharmacy, Aichi Gakuin University, Nagoya, Japan \\ 6)Laboratory of Medical Resources, School of Pharmacy, Aichi Gakuin University, Nagoya, Japan
}

(Received September 20, 2017; Accepted October 14, 2017)

\begin{abstract}
Dental pulp is known to play crucial roles in homeostasis of teeth and periodontal tissue. Although resorption of bone around the roots of nonvital teeth is occasionally observed in clinical practice, little is known about the role of dental pulp in osteoclastogenesis. Here we evaluated the effects of conditioned medium (CM) from rat dental pulp on osteoclastogenesis. It was found that the $\mathrm{CM}$ reduced the number of tartrate-resistant acid phosphatase (TRAP)-positive multinucleated osteoclasts, but did not alter the mRNA levels of nuclear factor of activated T-cells, cytoplasmic 1 and TRAP. To further understand the mechanism behind these results, we evaluated the effects of $\mathrm{CM}$ on osteoclast precursors and found that the CM removed cell processes, resulting in a significant reduction in the number of
\end{abstract}

Correspondence to Dr. Kazunori Hamamura, Department of Pharmacology, School of Dentistry, Aichi Gakuin University, 1-100 Kusumoto-cho, Chikusa-ku, Nagoya, Aichi 464-8650, Japan Fax:+81-52-752-5988 E-mail: hamak@dpc.agu.ac.jp

J-STAGE Advance Publication: July 9, 2018

Color figures can be viewed in the online issue at J-STAGE.

doi.org/10.2334/josnusd.17-0342

DN/JST.JSTAGE/josnusd/17-0342 attached cells and an increase in the number of freely floating cells. Furthermore, the CM suppressed the mRNA levels of focal adhesion kinase and paxillin, which are involved in cell adhesiveness and spreading. Collectively, the present results show that $\mathrm{CM}$ from dental pulp serves as an inhibitor of osteoclastogenesis by reducing the number and adhesiveness of osteoclast precursors, suggesting novel therapeutic applicability for osteoporosis.

Keywords: rat dental pulp; osteoclast precursors; osteoclasts; adhesiveness.

\section{Introduction}

Dental pulp is surrounded and sealed by dentine, enamel, and cementum, and this airtightness contributes to protecting dental pulp from bacteria in the oral environment $(1,2)$. Once hard dental tissues are destroyed by caries, bacteria may invade into the dental pulp, resulting in its inflammation (3). If this inflammatory situation continues, the dental pulp may become nonvital, followed by bone resorption around its root (4).

In dental pulp, genes such as calcitonin gene-related peptide, transient receptor potential vanilloid-1 (TRPV1), 
matrix metalloproteinase-9 (MMP-9), and vascular endothelial growth factor receptors are known to be expressed (5-9). Several studies have shown that these genes are involved in development of periapical lesions (7-9). For instance, ablation of TRPV1-expressing neurons in dental pulp results in destruction of periapical bone (8). It is also reported that periapical lesions in MMP9-knockout mice are larger than those in wild-type mice (7). Furthermore, a blocking antibody against VEGFR-2 in mice with periapical lesions induced by the pulp exposure promotes the extent of the lesions (9).

Dental pulp is composed of various cells, extracellular matrix, nerves, and capillaries. Most of the cells are fibroblasts (1), but immune cells such as macrophages, dendritic cells, and lymphocytes are also present. Macrophages can differentiate into multinucleated osteoclasts in the presence of receptor activator of nuclear factor kappa-B ligand (RANKL) and macrophage colonystimulating factor (M-CSF). However, little is known about the role of the dental pulp in osteoclastogenesis.

In this study, we prepared conditioned medium (CM) from dental pulp and examined its effects on osteoclastogenesis. Osteoclast precursors were cultured with RANKL in the presence and absence of the CM, and tartrate-resistant acid phosphatase (TRAP) staining was conducted to identify multinucleated osteoclasts. To evaluate the effects of the $\mathrm{CM}$ on osteoclast precursors, the cells were cultured in the presence and absence of the $\mathrm{CM}$ without RANKL, and the numbers of attached and freely floating cells were determined. To assess the adhesiveness of osteoclast precursors in the CM, the mRNA levels of focal adhesion kinase (FAK) and paxillin were determined using quantitative real-time PCR.

\section{Materials and Methods}

\section{Animals}

For harvesting of bone marrow cells and isolation of dental pulp, Wistar rats were purchased from Chubu Kagaku Shizai (Nagoya, Japan). Thirty-one male rats were used in this study. The protocols for animal experiments were approved by the Aichi Gakuin University Animal Research Committee (approval number: AGUD320).

\section{Cell culture}

Male Wistar rat bone marrow cells, isolated from long bones (femur and tibia), were cultured in $\alpha$ MEM containing $10 \%$ fetal bovine serum and antibiotics (Wako, Osaka, Japan). Cells were maintained at $37^{\circ} \mathrm{C}$ with $5 \% \mathrm{CO}_{2}$ in a humidified incubator. They were then stained with trypan blue and the numbers of live and dead cells were counted using a hemacytometer.

\section{Preparation of osteoclast precursors from bone marrow cells and osteoclastogenesis}

Primary osteoclast precursors were prepared as described previously (10). In brief, bone marrow cells were cultured with $10 \mathrm{ng} / \mathrm{ml}$ M-CSF (PeproTech, Rocky Hills, NC, USA) for 3 days, and the surface-attached cells were used as osteoclast precursors. For osteoclastogenesis, these cells were cultured with $10 \mathrm{ng} / \mathrm{ml} \mathrm{M}$-CSF and 50 ng/ml RANKL (PeproTech).

\section{Preparation of CM from rat dental pulp and tail tissue}

The rats were killed by cervical dislocation, and their maxillary incisors were extracted for isolation of the dental pulp. The tails excluding the skin were cut at the same length as the dental pulps. These tissues were then cultured in aMEM containing 10\% fetal bovine serum and antibiotics for 3 days. The culture medium was collected and centrifuged at 1,000 rpm for $5 \mathrm{~min}$, and the supernatants were collected and passed through a $0.22-\mu \mathrm{m}$ pore filter (Millipore, Darmstadt, Germany). The filtrate was used as CM in the various experiments.

\section{TRAP staining}

After culture of osteoclast precursors from bone marrow cells for $72 \mathrm{~h}$ with RANKL in the presence and absence of $\mathrm{CM}$, the cells were fixed in $10 \%$ formalin neutral buffer solution and incubated in sodium acetate buffer $(0.1 \mathrm{M}, \mathrm{pH} 5)$ containing naphthol AS-MX phosphate, Fast Red Violet LB Salt, and $\mathrm{MnCl}_{2}$ in the presence of sodium tartrate at $37^{\circ} \mathrm{C}$ for $60 \mathrm{~min}$ for TRAP staining. The number of TRAP-positive cells containing three or more nuclei was determined.

\section{Quantitative real-time PCR}

Total RNA was extracted using an RNeasy Plus mini kit and an RNeasy Micro kit (Qiagen, Germantown, MD, USA). Reverse transcription was conducted with a high-capacity cDNA reverse transcription kit (Applied Biosystems, Carlsbad, CA, USA), and quantitative realtime PCR was performed using TaKaRa Thermal Cycler Dice Real Time System III with THUNDERBIRD SYBR qPCR mix kits (TOYOBO, Osaka, Japan). The PCR cycling conditions were $95^{\circ} \mathrm{C}$ for $10 \mathrm{~min}$ for predenaturation, 40 cycles at $95^{\circ} \mathrm{C} / 15 \mathrm{~s}$ for denaturation, and $60^{\circ} \mathrm{C} / 1 \mathrm{~min}$ for extension. We evaluated the mRNA levels of nuclear factor of activated T-cells, cytoplasmic 1 (NFATc1), TRAP, FAK, and paxillin using the PCR primers listed in Table 1. GAPDH was used as an internal control. 
Table 1 Real-time PCR primers for mRNAs

\begin{tabular}{lll}
\hline Gene & Forward primer & Reverse primer \\
\hline FAK & 5'-CGTGAAGCCTTTTCAAGGAG -3' & 5'- TCCATCCTCATCCGTTCTTC -3' \\
paxillin & 5'- CCAGCACCAAAAATTCCAGT -3' & 5'-GGTCCAGCTCAGAGAGGTTG -3' \\
NFATc1 & 5'-ACGATGTGGAGGTGGAAGAC -3' & 5'-GGACGCCTCAGAGTTACAGC -3' \\
TRAP & 5'-GAGAACGGTGTGGGCTATGT -3' & 5'-GTGAAGCCACCCAGAGAGTC -3' \\
GAPDH & 5'-ATGACTCTACCCACGGCAAG -3' & 5'- TACTCAGCACCAGCATCACC -3' \\
\hline
\end{tabular}

A

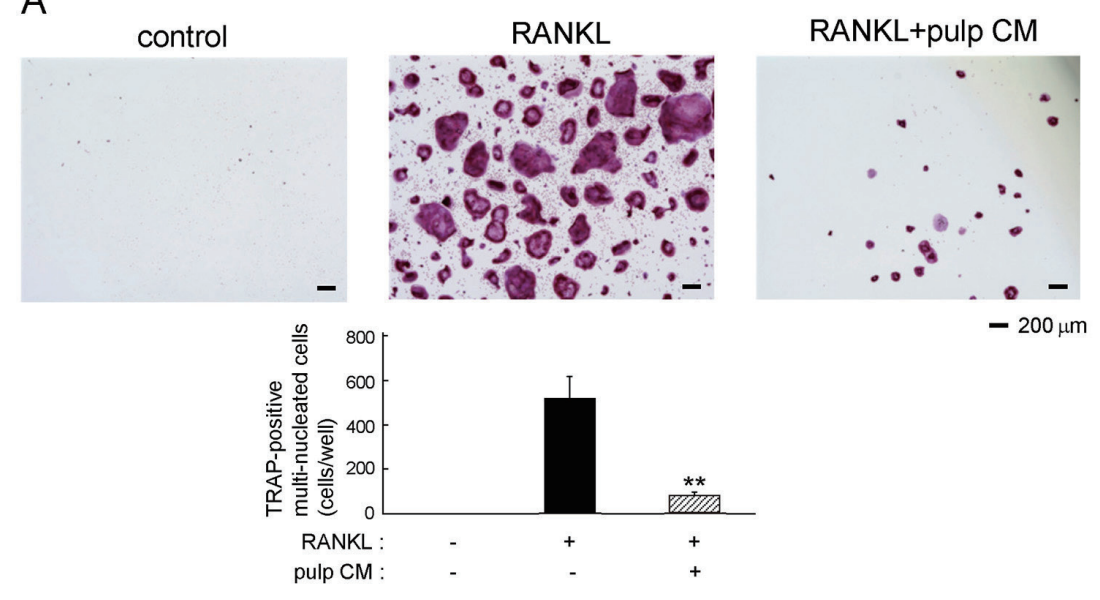

B
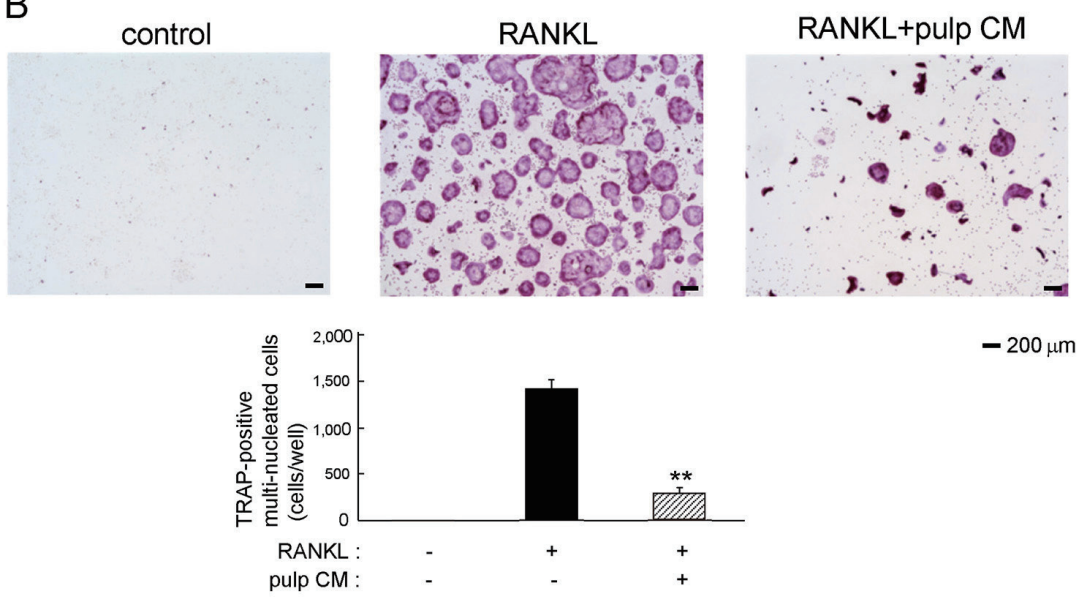

Fig. 1 Inhibitory effects of dental pulp CM on osteoclastogenesis. We cultured osteoclast precursors with 50 $\mathrm{ng} / \mathrm{mL}$ RANKL and dental pulp CM for $72 \mathrm{~h}$. (A) Suppression of differentiation of TRAP-positive multinucleated osteoclasts from osteoclast precursors by dental pulp CM $(n=6)$. Note that the RANKL administration group was also treated with $10 \% \mathrm{FBS}$, and that the RANKL plus CM group was also treated with $2 \%$ FBS and the remaining FBS in the CM. (B) Suppression of differentiation of TRAP-positive multinucleated osteoclasts from osteoclast precursors by dental pulp $\mathrm{CM}(n=6)$. Note that the RANKL administration group was also treated with $10 \%$ FBS, and that the RANKL plus CM group was also treated with $10 \%$ FBS and the remaining FBS in the CM. Data are expressed as mean \pm S.D. The double asterisks indicate $P<0.01$.

\section{Statistical analysis}

Three to six independent experiments were conducted, and data were expressed as mean \pm S.D. Statistical significance was evaluated using Student's $t$-test at $P<$ 0.05 . The single and double asterisks indicate $P<0.05$ and $P<0.01$, respectively.

\section{Results}

Inhibitory effects of dental pulp CM on osteoclastogenesis

The primary aim of this study was to evaluate the effects of dental pulp CM on osteoclastogenesis. We employed TRAP staining for the RANKL group (10\% fetal bovine serum - FBS) and the RANKL \& dental pulp CM group 

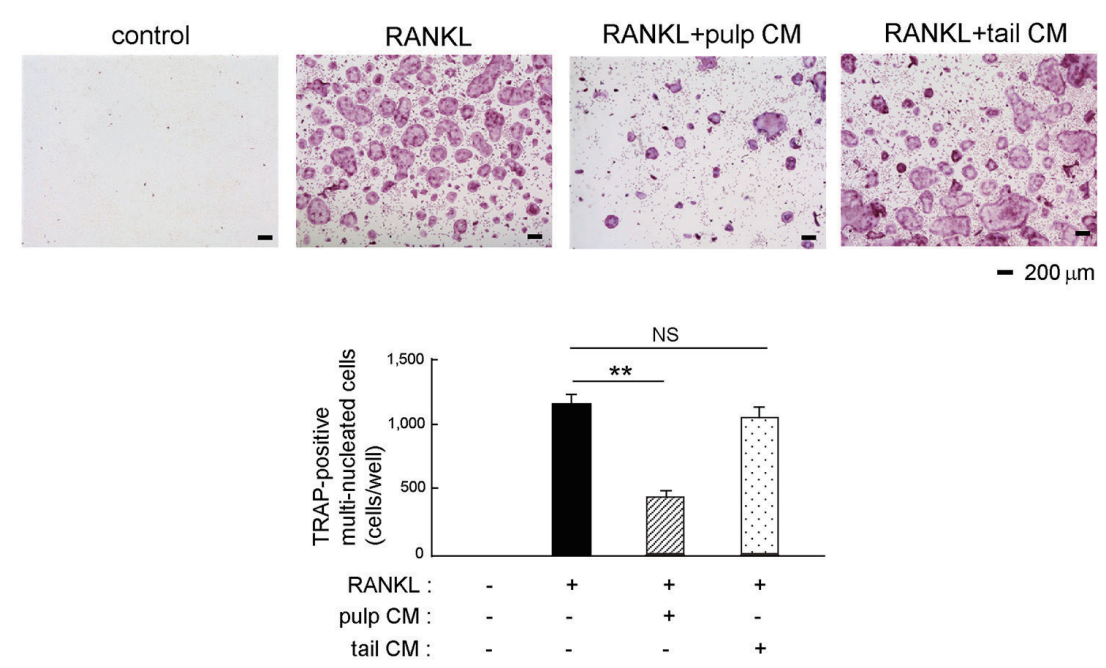

Fig. 2 Lack of apparent effects of CM derived from tail tissue on osteoclastogenesis. We cultured osteoclast precursors with $50 \mathrm{ng} / \mathrm{ml}$ RANKL and dental pulp or tail CM for $72 \mathrm{~h}$. The CM from the tail did not reduce the number of RANKL-induced TRAP-positive multinucleated osteoclasts $(n=5)$. Data are expressed as mean \pm S.D. The double asterisks indicate $P<0.01$. NS indicates no significance.
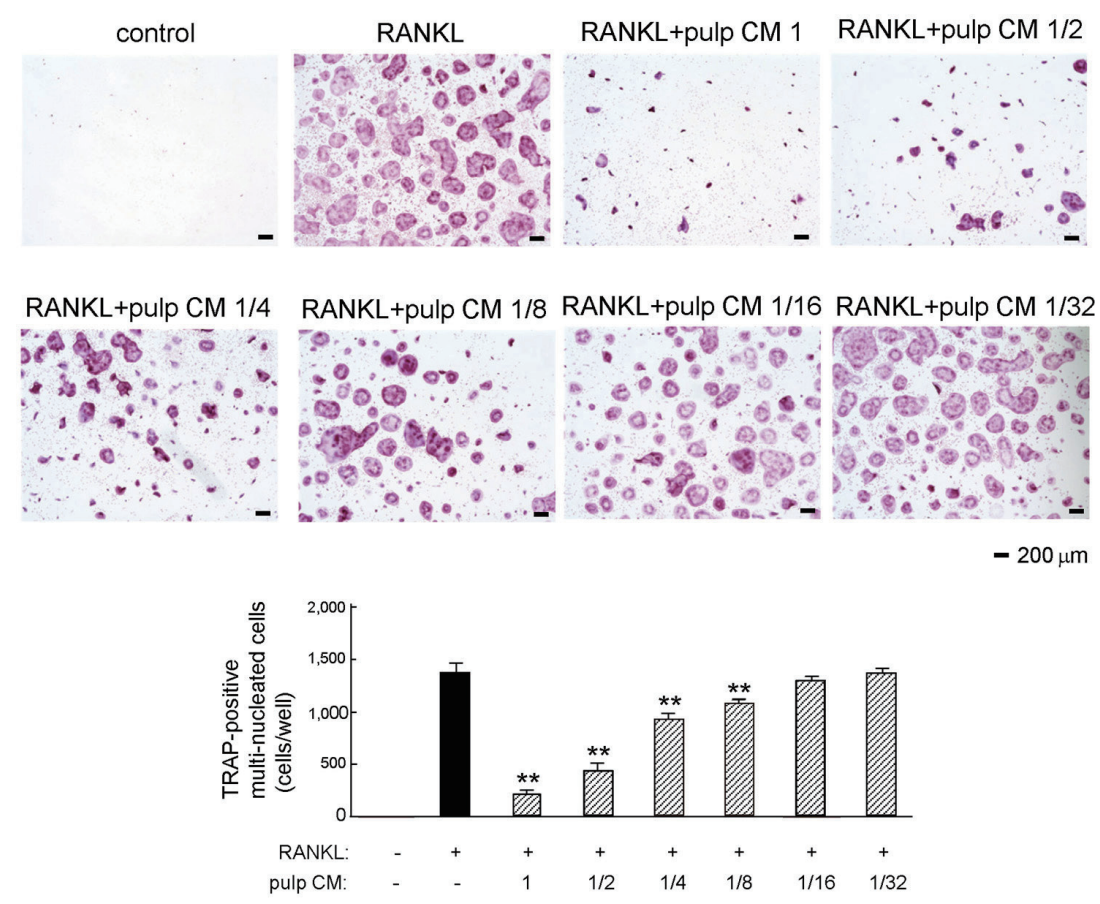

Fig. 3 Dose-dependent reduction in the number of TRAP-positive multinucleated osteoclasts by culture with dental pulp CM for $72 \mathrm{~h}$ (concentration of CM: 1 -fold to $1 / 32$-fold) $(n=3)$. Data are expressed as mean \pm S.D. The double asterisks indicate $P<0.01$.

( $2 \%$ or $10 \%$ FBS plus remaining FBS in the CM). The results showed that the $\mathrm{CM}$ reduced the number of TRAP-positive multinucleated osteoclasts (Fig. 1). In subsequent experiments, we used the RANKL group (10\% FBS) and the RANKL \& CM group (10\% FBS and remaining FBS in the $\mathrm{CM}$ ). To evaluate whether the observed inhibitory effect of the dental pulp CM is specific, we also employed CM derived from the tail tissue. The number of TRAP-positive multinucleated osteoclasts was significantly reduced by the dental pulp CM, but not by the tail CM (Fig. 2). The number of multinucleated osteoclasts was significantly reduced by the dental pulp CM at various doses (1-fold to 1/8-fold) in a dose-dependent manner (Fig. 3). 

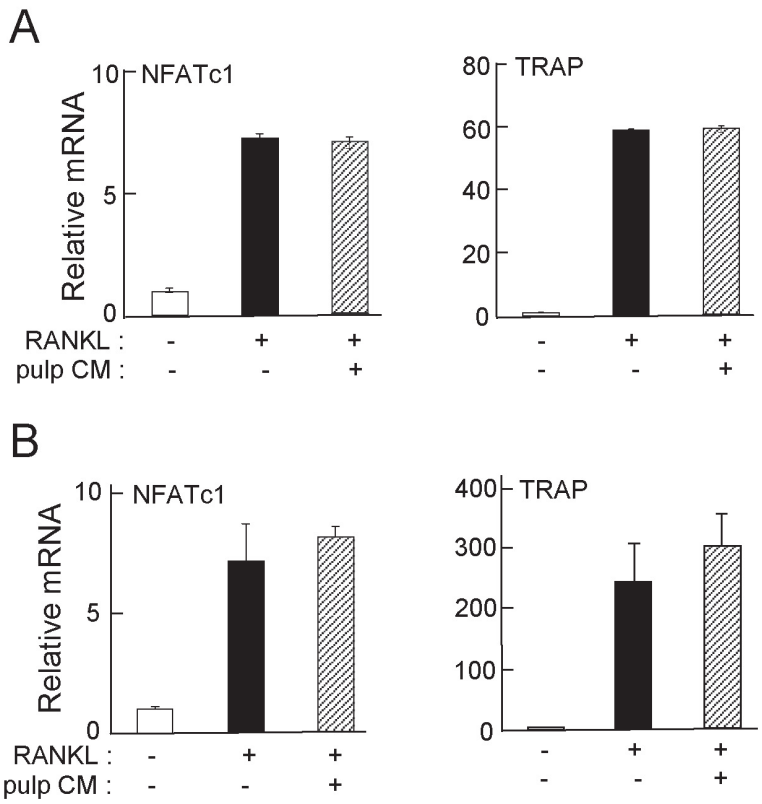

Fig. 4 Lack of apparent effects of dental pulp CM on the mRNA levels of NFATc1 and TRAP. (A) mRNA levels of NFATc1 and TRAP at $48 \mathrm{~h}$ after RANKL administration $(n=4)$. (B) mRNA levels of NFATc1 and TRAP at $72 \mathrm{~h}$ after RANKL administration $(n=4)$. Data are expressed as mean \pm S.D.

\section{Lack of detectable effects of dental pulp CM on NFATc1 and TRAP}

Although the dental pulp CM reduced the number of TRAP-positive multinucleated osteoclasts, it did not alter the levels of mRNA for NFATc1 and TRAP induced by RANKL (Fig. 4).

\section{Reduction in the number of osteoclast precursors and} attenuation of their adhesiveness by dental pulp CM To clarify the mechanism responsible for the reduction in the number of TRAP-positive multinucleated osteoclasts without any alteration in the expression of mRNAs for NFATc1 and TRAP, we evaluated the effects of dental pulp CM in the absence of RANKL. Dental pulp CM without RANKL significantly reduced the number of cells with processes (Fig. 5A, B). It also increased the number of freely floating cells and decreased the number of cells attached to the surface (Fig. 5C). However, there was no significant difference in the number of dead cells (Fig. 5D). Furthermore, the CM attenuated the levels of mRNA for FAK and paxillin, which are known to regulate cell adhesion and spreading (Fig. 5E).

\section{Discussion}

We demonstrated in this study that CM derived from dental pulp reduced the number of TRAP-positive multinucleated osteoclasts. In spite of the reduction in TRAP-positive multinucleated osteoclasts by dental pulp
$\mathrm{CM}$, the CM did not alter the mRNA levels of NFATc1 and TRAP. These results suggest that the CM did not affect osteoclast differentiation directly, but reduced the number of osteoclast precursors and attenuated their adhesiveness, resulting in the reduced number of multinucleated cells. Our results strongly indicate that the observed reduction in the number of multinucleated cells in response to dental pulp CM is not attributable to factors such as the serum concentration in the medium, which are not directly linked to dental pulp. To eliminate any possibility of serum-driven effects on osteoclastogenesis, we prepared two CM groups: $2 \%$ FBS plus remaining FBS in the CM, and 10\% FBS plus remaining FBS in the CM (Fig. 1A, B, respectively). In both groups, the $\mathrm{CM}$-driven reduction in the number of TRAP-positive multinucleated osteoclasts was observed regardless of the FBS concentration.

The CM derived from dental pulp reduced the expression of mRNAs for FAK and paxillin in osteoclast precursors. FAK is known to be involved in cell adhesion, proliferation and cytoskeletal rearrangement in various cells (11-15). For instance, it is reported that knockdown of FAK in osteoblastic MC3T3-E1 cells caused them to adopt a round morphology and attenuated their proliferation, adhesion, migration, and differentiation (15). Myeloid-specific FAK-knockout mice revealed loss of FAK, resulting in reduction of bone resorption by osteoclasts (14). Furthermore, Owen et al. reported that 
A

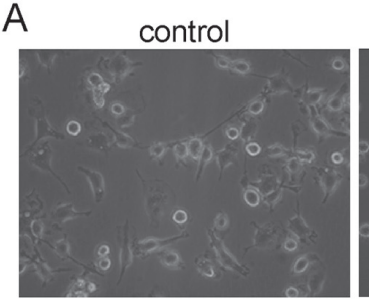

C

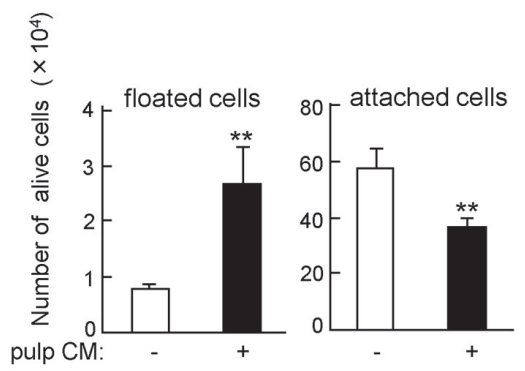

pulp CM

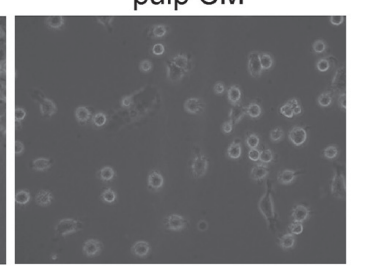

$\mathrm{D}$
B
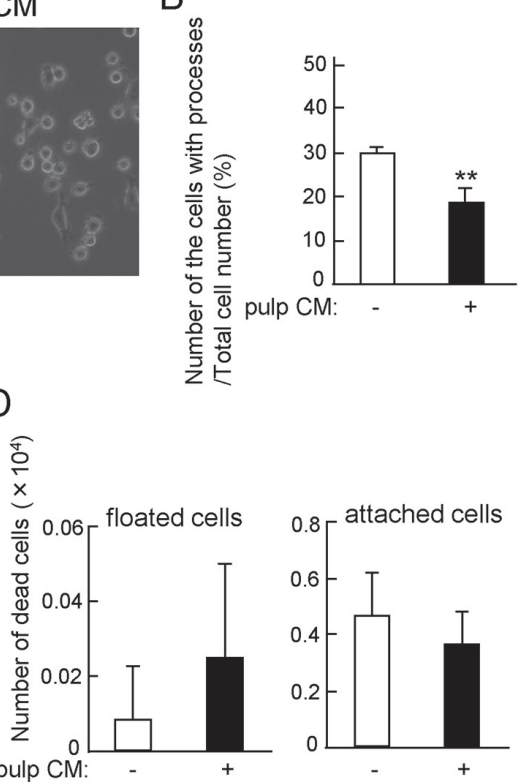

E
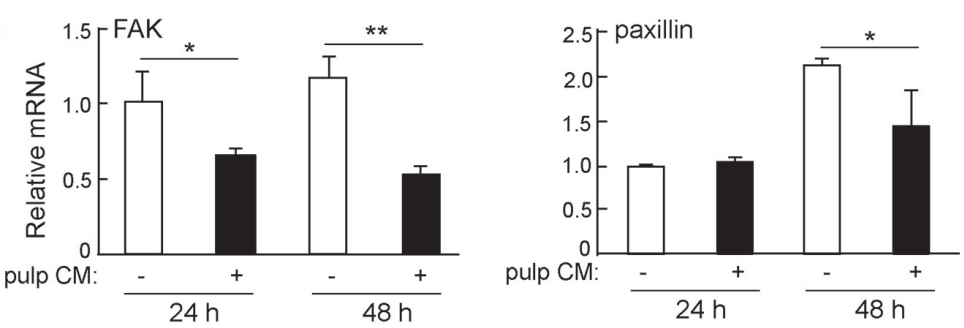

Fig. 5 Reduction in the number of osteoclast precursors and attenuation of their adhesiveness by dental pulp CM. We cultured osteoclast precursors with $10 \mathrm{ng} / \mathrm{mL}$ M-CSF and dental pulp CM (1/2-fold) for 24-72 h. (A) Disappearance of cell processes in osteoclast precursors upon culture with dental pulp CM. (B) Ratio of the number of the cells with processes after culture with dental pulp CM for $72 \mathrm{~h}(n=3)$. (C) Numbers of viable cells, both floating freely and attached, after culture with dental pulp CM for $72 \mathrm{~h}(n=3)$. (D) Numbers of dead cells, both floating freely and attached, after culture with dental pulp CM for $72 \mathrm{~h}(n=3)$. (E) Effects of culture with dental pulp CM for 24 and $48 \mathrm{~h}$ on expression of FAK and paxillin mRNAs $(n=4)$. Data are expressed as mean \pm S.D. The single and double asterisks indicate $P<0.05$ and $P<0.01$, respectively.

FAK regulates lamellipodium formation, adhesion, and motility in macrophages (12). Paxillin is also known to regulate cell migration, adhesion, and spreading (16-20). It is reported that paxillin regulates actin reorganization (16), and enhances cell spreading and stretching in macrophages (20). It is also reported that paxillin is required for organization of podosome rings in osteoclasts (18). Collectively, these studies suggest that dental pulp CM may reduce cell adhesion via downregulation of FAK and paxillin in osteoclast precursors.

Several studies have reported interactions of macrophages with dental pulp cells and stem cells (DPSCs) (21-24). However, it is still unclear whether co-cultured macrophages and dental pulp cells/DPSCs can produce an inflammatory response. Yonehiro et al. reported that co-culture of immortalized dental pulp cells and macrophages upregulated the production of inflammatory cytokines such as interleukin-6 (IL-6) and monocyte chemoattractant protein-1 (MCP-1) in response to lipopolysaccharide (LPS) stimulation (22). In contrast, Lee et al. reported that co-culture of DPSCs and macrophages markedly attenuated the secretion of tumor necrosis factor $\alpha$ (TNF- $\alpha$ ) in response to LPS (23). It has also been reported that CM from DPSCs increased the M2 polarization of M1 macrophages that had been activated by LPS, resulting in an increase of anti-inflammatory cytokines (24). These reports suggest that the immune responses of macrophages in response to LPS may depend on the presence of other cells. Furthermore, it has been reported that RANKL is expressed in dental pulp cells, and that co-culture of $\mathrm{CD} 14+$ monocytes derived from human peripheral blood and dental pulp cells promotes 
the number of osteoclasts via RANKL-RANK signaling (21). The present results suggest that the direct and indirect interactions of dental pulp cells with macrophages have differential effects on osteoclastogenesis.

Many studies have reported that the CM from DPSCs and human exfoliated deciduous teeth provides a powerful tool for investigating bone and dental pulp tissue regeneration, focal cerebral ischemia, and diabetes mellitus (25-29). Moreover, the factors secreted from DPSCs have been analyzed $(26,29,30)$. TGF- $\beta 1$ and FGF- 2 in the CM from DPSCs have been reported to counteract cytotoxicity induced by the base resin TEGDMA (26). Bronckaers et al. have reported that the CM from DPSCs contains various angiogenesis-related molecules such as vascular endothelial growth factor (VEGF), MCP-1, plasminogen activator inhibitor-1 (PAI-1), and endostatin (30). Piva et al. have also reported that the CM from DPSCs contains the angiogenic proteins endothelin, insulin-like growth factor binding protein-3 (IGFBP-3), pentraxin3, serpin E1, serpin F1, thrombospondin 1, tissue inhibitor of metalloproteinase-1, and VEGF (29). IGFBP-3 is reported to inhibit cell adhesion through downregulation of FAK in human umbilical vein endothelial cells (31). Although the present study revealed that dental pulp CM suppressed cell adhesion and reduced the mRNA levels of FAK and paxillin, further analysis will be necessary to identify the specific molecules in CM that are responsible for the down-regulation of FAK and paxillin, and suppression of cellular adhesion.

We also found that dental pulp CM reduced the number of TRAP-positive multinucleated osteoclasts due to attenuation of osteoclast precursor adhesiveness. Therefore, CM from the dental pulp of extracted teeth might be a potentially useful therapeutic option for osteoporosis if administered systemically.

\section{Acknowledgments}

We thank Dr. Hiroki Yokota, Indiana University Purdue University, Indianapolis, for critical reading of the manuscript. This study was supported in part by Grants-in-Aid for Scientific Research to Kazunori Hamamura (17K11657) from the Ministry of Education, Culture, Sports, Science and Technology, Japan.

\section{Conflict of interest}

The authors have no conflict of interest to declare.

\section{References}

1. Goldberg M, Smith AJ (2004) Cells and extracellular matrices of dentin and pulp: a biological basis for repair and tissue engineering. Crit Rev Oral Biol Med 15, 13-27.

2. Goldberg M, Hirata A (2017) The dental pulp: composition, properties and functions. JSM Dent 5, 1079.

3. Jontell M, Okiji T, Dahlgren U, Bergenholtz G (1998) Immune defense mechanisms of the dental pulp. Crit Rev Oral Biol Med 9, 179-200.

4. Bergenholtz G (1990) Pathogenic mechanisms in pulpal disease. J Endod 16, 98-101.

5. Casasco A, Calligaro A, Casasco M, Springall DR, Polak JM, Poggi P et al. (1990) Peptidergic nerves in human dental pulp. An immunocytochemical study. Histochemistry 95, 115-121.

6. Gibbs JL, Melnyk JL, Basbaum AI (2011) Differential TRPV1 and TRPV2 channel expression in dental pulp. J Dent Res 90, 765-770.

7. Wan C, Yuan G, Yang J, Sun Q, Zhang L, Zhang J et al. (2014) MMP9 deficiency increased the size of experimentally induced apical periodontitis. J Endod 40, 658-664.

8. Austah ON, Ruparel NB, Henry MA, Fajardo RJ, Schmitz JE, Diogenes A (2016) Capsaicin-sensitive innervation modulates the development of apical periodontitis. J Endod 42, 1496-1502.

9. Virtej A, Papadakou P, Sasaki H, Bletsa A, Berggreen E (2016) VEGFR-2 reduces while combined VEGFR-2 and -3 signaling increases inflammation in apical periodontitis. $\mathrm{J}$ Oral Microbiol 8, 32433.

10. Hamamura K, Chen A, Tanjung N, Takigawa S, Sudo A, Yokota H (2015) In vitro and in silico analysis of an inhibitory mechanism of osteoclastogenesis by salubrinal and guanabenz. Cell Signal 27, 353-362.

11. Zachary I, Rozengurt E (1992) Focal adhesion kinase (p125FAK): a point of convergence in the action of neuropeptides, integrins, and oncogenes. Cell 71, 891-894.

12. Owen KA, Pixley FJ, Thomas KS, Vicente-Manzanares M, Ray BJ, Horwitz AF et al. (2007) Regulation of lamellipodial persistence, adhesion turnover, and motility in macrophages by focal adhesion kinase. J Cell Biol 179, 1275-1287.

13. Hamamura K, Tsuji M, Ohkawa Y, Nakashima H, Miyazaki $\mathrm{S}$, Urano $\mathrm{T}$ et al. (2008) Focal adhesion kinase as well as p130Cas and paxillin is crucially involved in the enhanced malignant properties under expression of ganglioside GD3 in melanoma cells. Biochim Biophys Acta 1780, 513-519.

14. Ray BJ, Thomas K, Huang CS, Gutknecht MF, Botchwey EA, Bouton AH (2012) Regulation of osteoclast structure and function by FAK family kinases. J Leukoc Biol 92, 10211028.

15. Horikiri Y, Shimo T, Kurio N, Okui T, Matsumoto K, Iwamoto $\mathrm{M}$ et al. (2013) Sonic hedgehog regulates osteoblast function by focal adhesion kinase signaling in the process of fracture healing. PLoS One 8, e76785.

16. Williams LM, Ridley AJ (2000) Lipopolysaccharide induces actin reorganization and tyrosine phosphorylation of Pyk2 and paxillin in monocytes and macrophages. J Immunol 164, 2028-2036.

17. Hagel M, George EL, Kim A, Tamimi R, Opitz SL, Turner $\mathrm{CH}$ et al. (2002) The adaptor protein paxillin is essential for normal development in the mouse and is a critical transducer of fibronectin signaling. Mol Cell Biol 22, 901-915. 
18. Badowski C, Pawlak G, Grichine A, Chabadel A, Oddou C, Jurdic P et al. (2008) Paxillin phosphorylation controls invadopodia/podosomes spatiotemporal organization. Mol Biol Cell 19, 633-645.

19. Shibuya H, Hamamura K, Hotta H, Matsumoto Y, Nishida Y, Hattori H et al. (2012) Enhancement of malignant properties of human osteosarcoma cells with disialyl gangliosides GD2/ GD3. Cancer Sci 103, 1656-1664.

20. St-Pierre J, Ostergaard HL (2013) A role for the protein tyrosine phosphatase CD45 in macrophage adhesion through the regulation of paxillin degradation. PLoS ONE 8, e71531.

21. Uchiyama M, Nakamichi Y, Nakamura M, Kinugawa S, Yamada H, Udagawa N et al. (2009) Dental pulp and periodontal ligament cells support osteoclastic differentiation. J Dent Res 88, 609-614.

22. Yonehiro J, Yamashita A, Yoshida Y, Yoshizawa S, Ohta K, Kamata N et al. (2012) Establishment of an ex vivo pulpitis model by co-culturing immortalized dental pulp cells and macrophages. Int Endod J 45, 1103-1108.

23. Lee S, Zhang QZ, Karabucak B, Le AD (2016) DPSCs from inflamed pulp modulate macrophage function via the TNF- $\alpha$ / IDO axis. J Dent Res 95, 1274-1281.

24. Omi M, Hata M, Nakamura N, Miyabe M, Kobayashi Y, Kamiya H et al. (2016) Transplantation of dental pulp stem cells suppressed inflammation in sciatic nerves by promoting macrophage polarization towards anti-inflammation phenotypes and ameliorated diabetic polyneuropathy. J Diabetes Investig 7, 485-496.

25. Inoue T, Sugiyama M, Hattori H, Wakita H, Wakabayashi T,
Ueda M (2013) Stem cells from human exfoliated deciduous tooth-derived conditioned medium enhance recovery of focal cerebral ischemia in rats. Tissue Eng Part A 19, 24-29.

26. Paschalidis T, Bakopoulou A, Papa P, Leyhausen G, Geurtsen W, Koidis P (2014) Dental pulp stem cells' secretome enhances pulp repair processes and compensates TEGMAinduced cytotoxicity. Dent Mater 30, e405-418.

27. Omori M, Tsuchiya S, Hara K, Kuroda K, Hibi H, Okido M et al. (2015) A new application of cell-free bone regeneration: immobilizing stem cells from human exfoliated deciduous teeth-conditioned medium onto titanium implants using atmospheric pressure plasma treatment. Stem Cell Res Ther $6,124$.

28. Izumoto-Akita T, Tsunekawa S, Yamamoto A, Uenishi E, Ishikawa K, Ogata H et al. (2015) Secreted factors from dental pulp stem cells improve glucose intolerance in streptozotocin-induced diabetic mice by increasing pancreatic $\beta$-cell function. BMJ open Diabetes Res care 3, e000128.

29. Piva E, Tarle SA, Nor JE, Zou D, Hatfield E, Guinn T et al. (2017) Dental pulp tissue regeneration using dental pulp stem cells isolated and expanded in human serum. J Endod 43, 568-574.

30. Bronckaers A, Hilkens P, Fanton Y, Struys T, Gervois P, Politis C et al. (2013) Angiogenic properties of human dental pulp stem cells. PLoS One 8, e 71104.

31. Lee HJ, Lee JS, Hwang SJ, Lee HY (2015) Insulin-like growth factor binding protein-3 inhibits cell adhesion via suppression of integrin $\beta 4$ expression. Oncotarget 6, 15150-15163. 